\title{
Superreplication of European multiasset derivatives with bounded stochastic volatility
}

\author{
Fausto Gozzi \\ Dipartimento di Matematica per le Decisioni \\ Economiche, Finanziarie e Assicurative \\ Università di Roma "La Sapienza" \\ Via del Castro Laurenziano 9 - I-00100 Roma (Italy) \\ email: gozzi@dm.unipi.it \\ Tiziano Vargiolu* \\ Dipartimento di Matematica Pura ed Applicata \\ Università di Padova \\ Via Belzoni 7 - I-35131 Padova (Italy) \\ email: vargiolu@math.unipd.it
}

January 11, 2002

\begin{abstract}
In this paper we analyze the superreplication approach in stochastic volatility models in the case of European multiasset derivatives. We prove that the Black-ScholesBarenblatt (BSB) equation gives a superhedging strategy even if its solution is not twice differentiable. This is done under convexity assumptions on the final payoff $h$ that are verified in some applications presented here.
\end{abstract}

Keywords: superreplication, stochastic volatility, stochastic optimal control, HamiltonJacobi-Bellman equations.

\section{Introduction}

The main purpose of this paper is to analyze the superreplication approach in stochastic volatility models for European multiasset derivatives, including also cases when the value function is nonsmooth. In particular, we show that if the final payoff is convex and the price process has marginal laws absolutely continuous with respect to the $n$-dimensional Lebesgue measure, then a superhedging strategy is given by the gradient of the solution of a nonlinear partial differential equation (PDE), called the Black-Scholes-Barenblatt (BSB) equation.

\footnotetext{
${ }^{*}$ corresponding author. Both the authors gratefully acknowledge financial support from the CNR Strategic Project Modellizzazione matematica di fenomeni economici. The second author acknowledge financial support from the Research Training Network DYNSTOCH, under the programme Improving Human Potential financed by the The Fifth Framework Programme of the European Commission.
} 
We first give an outline of the problem with a formulation that uses forward prices (see e.g. [8]). We have a riskless asset, whose value we suppose to be constant through time, and $n$ risky assets whose prices $S=\left(S^{1}, \ldots, S^{n}\right)$ are governed by the dynamics

$$
d S_{t}=\bar{S}_{t} \sigma_{t} d W_{t},
$$

where $W$ is a $d$-dimensional Brownian motion under a so-called forward-neutral measure $\mathbb{Q}$, the matrix process $\sigma$ is adapted and takes values in a closed bounded set $\Sigma \subset M(n, d, \mathbb{R})$, and $\bar{S}_{t}=\operatorname{diag}\left(S_{t}\right)$. We then consider an agent who wants to hedge a European contingent claim whose payoff is a deterministic function $h(\cdot)$ which is globally Lipschitz continuous, calculated in $S_{T}$. Since the market could be incomplete because of the stochastic volatility $\sigma$ and the agent is not able to hedge the volatility, he chooses to hedge the option by using the superhedging approach. Following [1], [17] and [20], he fixes an initial capital at time $t$ as $V_{t}=V\left(t, S_{t}\right)$ and builds a self-financing portfolio consisting of a quantity $\Delta_{t}^{i}=\frac{\partial V}{\partial S_{i}}\left(t, S_{t}\right)$ (a so-called Markov superhedging strategy) of the asset $S^{i}, i=1, \ldots, n$, where $V(t, s)$ is the solution of a nonlinear PDE, similar to the Black-Scholes equation, called the Black-ScholesBarenblatt (BSB) equation in analogy with [1] and [20]. This equation is a Hamilton-JacobiBellman equation and it is linked to a stochastic control problem that has a nice financial interpretation as a "game against the market". The BSB equation is in general nonlinear (only in particular cases does it reduce to a linear Black-Scholes equation, see Section 5.1 and [20, Section 8]), and its use also appears in more general situations (see e.g. [6, 21], where the authors deal with constraints on the superhedging strategy and on its Gamma with a particular choice of the volatility).

The procedure depicted above works if the BSB equation has a $C^{1,2}$ regular solution (i.e. $C^{1}$ in time and $C^{2}$ in space), as is shown in $[17,20]$. This regularity is typically implied by a mathematical condition called uniform parabolicity, that in our model depends essentially on the set $\Sigma$ being composed of full rank matrices. This is not verified in some financial examples (see Example 12 for a typical situation). For this reason it is interesting to see what can be said in non uniformly parabolic cases (see Subsections 5.3 and 5.4 for examples).

In this paper, which is an extension of a previous work [20] by one of the authors, we give a partial answer to the problem of whether there could be a Markov superhedging strategy when the BSB equation is not uniformly parabolic. In particular, in the general case the BSB equation admits a unique solution $V$ with first derivatives defined almost everywhere and we show that $\Delta_{t}^{i}=\frac{\partial V}{\partial S_{i}}\left(t, S_{t}\right), i=1, \ldots, n, t \in[0, T]$, is a superhedging strategy when the payoff $h$ is convex and the random variables $S_{t}$ have a density with respect to the $n$ dimensional Lebesgue measure. This generalizes the classical result to some non-smooth cases.

The picture is still not complete, since we are not able, up to now, to show that the strategy $\Delta_{t}^{i}=\frac{\partial V}{\partial S_{i}}\left(t, S_{t}\right), i=1, \ldots, n, t \in[0, T]$, is a superstrategy also in the case of non convex payoff $h$. This happens in some particular cases, as the example in Subsection 5.4 shows, and the general problem is at the moment open.

From the technical point of view we observe that to get existence, uniqueness and regularity results for the BSB equation we need to use the theory of viscosity solutions. This part is taken from a paper of one of the two authors [23], whose results we recall in Section 3 for the reader's convenience. On the other hand, to prove that $\Delta_{t}=\frac{\partial V}{\partial S_{i}}\left(t, S_{t}\right)$ is a superreplicating strategy we need to use an approximation technique with compactness arguments that holds when $h$ is convex and the marginal laws of $S_{t}$ are absolutely continuous with respect to the Lebesgue measure for all $t \in[0, T]$. In order to get this result, we approximate the solution $V$ of the non-uniformly parabolic BSB equation (3) with a sequence 
$\left(V_{\varepsilon_{n}}\right)_{n}$, where $V_{\varepsilon}$ is the solution of a BSB equation having $\Sigma_{\varepsilon}=\Sigma+\varepsilon I$ and such that the equation is uniformly parabolic. Then the functions $V_{\varepsilon}$ converge pointwise to $V$, because they are all value functions of suitable optimal control problems. Moreover, by using an estimate on the weak first derivatives of $V_{\varepsilon}$ and $V$ based on estimates on the function $h$, together with the fact that the $V_{\varepsilon}$ and $V$ are convex (thanks to the particular structure of the control problem), we obtain a compactness result in $W_{\text {loc }}^{1, p}$, that allows us to obtain a sequence $\left(V_{\varepsilon_{n}}\right)_{n}$ such that $D_{s} V_{\varepsilon_{n}}$ converges in $L^{p}$ to $D_{s} V$. This convergence result implies that also the hedging portfolio $\Pi_{t}$ is the limit of portfolios $\Pi_{t}^{n}$ defined via the functions $V_{\varepsilon_{n}}$. Since the portfolios of the approximating problems are all superhedging, so too is the limit portfolio, and we have our result.

The paper is organized as follows: in Section 2 we present the problem, and for the reader's convenience we recall Theorem 5 that states that the strategy defined above via the BSB equation is a superreplicating one if the BSB equation has a smooth solution. In Section 3 we present the results on the BSB equation that we need later; in Section 4 we prove the limit theorems about the price and the hedging portfolio, and we obtain that the portfolio defined via the BSB equation is superhedging also with weak smoothness; in Section 5 we present some examples of progressively increasing complexity which use the theory developed in Section 3 and 4.

We wish to thank M. Bardi, L. Caffarelli, Yu. Kabanov, N. V. Krylov, M. Pratelli, W. J. Runggaldier, A. Święch and J. Zabczyk for many useful discussions on various points of this work.

\section{The model}

We suppose that there exists a riskless asset $M$ and $n$ risky assets $S^{i}, i=1, \ldots, n$ in the market. We make the usual assumptions that there exist a probability space $(\Omega, \mathcal{F}, \mathbb{P})$, a complete and right-continuous filtration $\left(\mathcal{F}_{t}\right)_{t \in[0, T]}$, where $\mathcal{F}_{t}$ represents the information available up to time $t$ and that $S^{i}$ are stochastic processes adapted to $\left(\mathcal{F}_{t}\right)_{t}$. Moreover, we assume that there exists a probability measure $\mathbb{Q}$, equivalent to $\mathbb{P}$, which is called forwardneutral probability [8], such that the value of the riskless asset $M$ remains constantly equal to 1 through time, and the dynamics of the prices of the assets $S^{i}$ under $\mathbb{Q}$ are the following:

$$
d S_{t}^{i}=S_{t}^{i}\left\langle\sigma_{t}^{i}, d W_{t}\right\rangle
$$

where $\left(W_{t}\right)_{t}$ is a $d$-dimensional $\mathbb{Q}$-Brownian motion adapted to $\left(\mathcal{F}_{t}\right)_{t},\langle\cdot, \cdot\rangle$ is the Euclidean scalar product in $\mathbb{R}^{d}, \sigma^{i}$ is a $d$-dimensional process for all $i=1, \ldots, n$, such that $\sigma=$ $\left(\sigma^{i}\right)_{i} \in \mathcal{A}(\Sigma)$, where $\Sigma$ is a closed bounded set in the space of $n \times d$ real matrices $M(n, d, \mathbb{R})$ and $\mathcal{A}(\Sigma)$ (which we call set of admissible volatilities) is the set of $\Sigma$-valued processes progressively measurable with respect to $\left(\mathcal{F}_{t}\right)_{t}$.

Remark 1 We do not assume that the market is complete. This can happen, roughly speaking, for various reasons:

- the filtration $\left(\mathcal{F}_{t}\right)_{t}$ can be strictly larger than the one generated by $W$ or than the one generated by $S$;

- information about the law of $\sigma$ may be not available;

- the number of Brownian motions $d$ can be greater than the number of assets $n$; moreover, even if $d \leq n$, the volatility matrix may be degenerate. 
Remark 2 The assumption that the interest rate is zero can be achieved by a so-called change of numeraire, that is by expressing the prices of all the assets in the market in units of a zero coupon bond with maturity $T$ (for more details, see [8]).

We can write the dynamics of the risky assets in a more compact vectorial notation as

$$
d S_{t}=\bar{S}_{t} \sigma_{t} d W_{t}
$$

where

$$
\bar{S}=\operatorname{diag}(S)=\left(\begin{array}{cccc}
S^{1} & 0 & \ldots & 0 \\
0 & \ddots & \ddots & \vdots \\
\vdots & \ddots & \ddots & 0 \\
0 & \ldots & 0 & S^{n}
\end{array}\right)
$$

We will also write $S^{\sigma}$ when we want to emphasize the dependence of $S$ on the particular volatility process $\sigma$.

Now we consider an agent in the market who does not know the true volatility $\sigma$ and needs to hedge a European derivative asset with payoff $h\left(S_{T}\right)=h\left(S_{T}^{1}, \ldots, S_{T}^{n}\right)$, where $h$ is a locally Lipschitz continuous function having polynomial growth.

Remark 3 In applied examples, the payoff $h$ is usually a globally Lipschitz continuous function so its growth is at most linear. We decided to work with the more general case of polynomially growing $h$ for expository reasons; in fact most of our results hold for such $h$. Later, when the global Lipschitz property of $h$ is needed, we will impose it as an extra assumption).

As usual, the agent can build a self-financing portfolio $\Pi$, holding $\eta_{t}$ units of the money market account $M$ and $\Delta_{t}^{i}$ units of the asset $S^{i}, i=1, \ldots, N$, at time $t$, where $\eta$ is adapted and the $\Delta^{i}, i=1, \ldots, N$, are progressively measurable with respect to $\left(\mathcal{F}_{t}\right)_{t}$. We indicate by $\Pi_{t}$ the value of the portfolio at time $t$, defined by

$$
\Pi_{t}=\eta_{t}+\left\langle\Delta_{t}, S_{t}\right\rangle
$$

where $\Delta_{t}=\left(\Delta_{t}^{1}, \ldots, \Delta_{t}^{n}\right)$. In order to prevent arbitrage opportunities, we assume that the portfolio is admissible and self-financing. We say that the portfolio is admissible if $\Pi$ is a supermartingale: if this happens, then $\Pi_{0}=0$ implies $\mathbb{E}\left[\Pi_{T}\right] \leq 0$. We say that the portfolio is self-financing if $\Pi$ obeys the dynamics

$$
d \Pi_{t}=\left\langle\Delta_{t}, d S_{t}\right\rangle
$$

If the portfolio is self-financing, then the initial value $\Pi_{0}$ and the process $\Delta$ are sufficient to characterize it, as $\Pi_{t}=\Pi_{0}+\int_{0}^{t}\left\langle\Delta_{t}, d S_{t}\right\rangle$ and $\eta_{t}=\Pi_{t}-\left\langle\Delta_{t}, S_{t}\right\rangle$. For this reason, if the portfolio is self-financing, we will also say that $\Delta$ is an admissible strategy if the resulting portfolio is a supermartingale.

The market can be not complete because of the unknown (possibly stochastic) volatility $\sigma$, so the agent cannot expect to replicate exactly any general contingent claim, and he has to choose some criterion to hedge the claim $h$. Assume that he chooses to use the superhedging approach, which we present in this situation. We define the superreplication capital for the contingent claim $h$ at time 0 as

$$
V^{+}\left(0, S_{0}\right)=\inf \left\{v \mid \forall \sigma \in \mathcal{A}(\Sigma) \exists \Delta \text { admissible s.t. } v+\int_{0}^{T}\left\langle\Delta_{t}, d S_{t}^{\sigma}\right\rangle \geq h\left(S_{t}^{\sigma}\right) \mathbb{P} \text {-a.s. }\right\}
$$


and define a superhedging strategy, or more briefly superstrategy, as any such process $\Delta$ (which possibly depends on $\sigma$ ). Similarly, we define the subreplication capital for $h$ at time 0 as

$$
V^{-}\left(0, S_{0}\right)=\sup \left\{v \mid \forall \sigma \in \mathcal{A}(\Sigma) \exists \Delta \text { admissible s.t. } v+\int_{0}^{T}\left\langle\Delta_{t}, d S_{t}^{\sigma}\right\rangle \leq h\left(S_{t}^{\sigma}\right) \mathbb{P} \text {-a.s. }\right\}
$$

and define a subhedging strategy, or more briefly substrategy, as any such process $\Delta$. If $V^{+}=V^{-}=V$, then $V$ is called the arbitrage free price of the contingent claim $h$ at time 0 .

Remark 4 We notice that this definition of super- and subreplication capital differs from the usual one in the literature (see e.g. $[6,10,21]$ ) by the presence of the unknown volatility. In fact, since the agent does not know the true volatility $\sigma$, he must protect himself against all the possible volatilities $\gamma \in \mathcal{A}(\Sigma)$. Notice that if $\gamma \neq \sigma$, then the laws of the price processes $S^{\gamma}$ and $S^{\sigma}$ can be mutually singular (see [19] for a detailed analysis in dimension 1). This does not contradict no-arbitrage: in fact, the absence of arbitrage is "morally" equivalent to the fact that $S^{\sigma}$ should have equivalent laws under $\mathbb{P}$ and $\mathbb{Q}$, not to the fact that $S^{\sigma}$ has laws equivalent to those of $S^{\gamma}$ (which are two different processes) under $\mathbb{P}$ or $\mathbb{Q}$. Since the law of $S^{\sigma}$ under $\mathbb{P}$ is equivalent to law of $S^{\sigma}$ under $\mathbb{Q}$, we can see that the definition of super- and subreplication capital is invariant with respect to the choice of the probability measure, as long as it is equivalent to $\mathbb{P}$ (which in general does not happen with other criteria, such as utility evaluation or minimization of risk measures). For this reason, we can work without problems under the forward neutral measure $\mathbb{Q}$.

In the above context, super- and substrategies depend on the particular volatility process $\sigma$, which is not known to the agent. For this reason, the agent will be interested in finding those which we call Markov super- (sub-)strategies, i.e. super- (sub-)strategies $\Delta$ of the type $\Delta_{t}=\Delta\left(t, S_{t}^{\sigma}\right)$, where $(t, s) \rightarrow \Delta(t, s)$ is a deterministic function. In this way, the strategy $\Delta$ will be calculated starting from quantities that are directly observable by the agent.

We notice that superstrategies and substrategies are good candidates to be arbitrages: in fact if we have (say) $\Pi_{T} \geq h\left(S_{T}\right) \mathbb{Q}$-a.s. and $\Pi_{T}>h\left(S_{T}\right)$ with positive probability, then the agent succeeds in making a profit with no initial endowment. This means that $V^{+}\left(0, S_{0}\right)$ has to be interpreted as an arbitrage upper bound for the price of the claim at time 0 ; similarly, the value $V^{-}\left(0, S_{0}\right)$ in a substrategy has to be interpreted as a lower bound. In fact, if the price of the claim is $V^{+}\left(0, S_{0}\right)$ or more, one could build an arbitrage by shortselling the claim and by buying the superhedging portfolio, thus making the final profit $\Pi_{T}-h\left(S_{T}\right) \geq 0$ with a negative initial investment. A similar argument applies to $V^{-}\left(0, S_{0}\right)$.

As already outlined in [1], [9], [17] and [20], natural candidates to be respectively superreplication capital and a Markov superstrategy in this context are

$$
V^{+}\left(0, S_{0}\right)=V\left(0, S_{0}\right), \quad \Delta_{t}=D_{s} V\left(t, S_{t}\right), \quad t \in[0, T]
$$

where $V$ is the solution of the following PDE:

$$
\begin{cases}\frac{\partial V}{\partial t}(t, s)+\frac{1}{2} \max _{\gamma \in \Sigma} \operatorname{tr}\left(D_{s}^{2} V(t, s)(\bar{s} \gamma)(\bar{s} \gamma)^{*}\right)=0, & t \in[0, T), s \in \mathbb{R}_{+}^{n} \\ V(T, s)=h(s), & s \in \mathbb{R}_{+}^{n}\end{cases}
$$


where $D_{s} V(t, s)=\left(\frac{\partial V}{\partial s_{1}}(t, s), \ldots, \frac{\partial V}{\partial s_{n}}(t, s)\right)$, and $D_{s}^{2} V(t, s)=\left(\frac{\partial^{2} V}{\partial s_{i} \partial s_{j}}(t, s)\right)_{i j}$. Equation (3), as in [1], will be called from now on the Black-Scholes-Barenblatt (BSB) equation for $h$. Moreover, in order to have a substrategy it is sufficient to proceed as above by substituting the maximum operator in Equation (3) with a minimum.

This choice has a stochastic control interpretation of a game against nature (which in this case is represented by the market). The agent does not know the volatility $\sigma$ (except for the fact that it takes values in $\Sigma$ ), but he assumes that the market plays against him. Then, in order to hedge the asset he can use the model

$$
d S_{t}^{\gamma}=\bar{S}_{t}^{\gamma} \gamma_{t} d W_{t}
$$

where the process $\gamma \in \mathcal{A}(\Sigma)$ is any admissible volatility. We can interpret the $\gamma$ as a control, corresponding to the subjective volatility of the agent, that he uses in order to decide his strategy. If the market were complete and the dynamics were given by Equation (4) with $\gamma$ known, then the arbitrage free price of the European contingent claim $h$ at time 0 would be

$$
V_{0}^{\mathbb{Q}, \gamma}=\mathbb{E}_{\mathbb{Q}}\left[h\left(S_{T}^{\gamma}\right)\right]
$$

where $\mathbb{Q}$ would be the unique forward-neutral measure of the problem. Since the market is not complete, we are not sure that the contingent claim defined by $h$ is attainable. In particular, the volatility $\gamma$ is not known. The agent then can use the following construction coming from stochastic optimal control. We define reference probability system $\nu$ as a 4-uple

$$
\nu=\left(\Omega,\left(\mathcal{F}_{u}\right)_{u \in[t, T]}, \mathbb{P}, W\right)
$$

where $\left(\Omega, \mathcal{F}_{T}, \mathbb{Q}\right)$ is a probability space, and $W$ is a Brownian motion adapted to the filtration $\left(\mathcal{F}_{u}\right)_{u}$. Let

$$
\mathcal{A}_{t, \nu}(\Sigma)=\{\gamma \mid \gamma \text { progressively measurable } \Sigma \text {-valued process defined on } \nu\}
$$

We consider the supremum of the prices among all $\gamma \in \mathcal{A}_{t, \nu}(\Sigma)$ :

$$
V_{\nu}^{+}(t, s)=\sup _{\gamma \in \mathcal{A}_{t, \nu}(\Sigma)} \mathbb{E}_{\mathbb{Q}}\left[h\left(S_{T}^{\gamma}\right)\right]
$$

and we define the value function to be the function $V(t, x)$ defined as

$$
V^{+}(t, x)=\sup _{\nu} V_{\nu}(t, x)
$$

In an analogous way, the agent can build another value function corresponding to the lowest possible price:

$$
V^{-}(t, x)=\inf _{\nu} \inf _{\gamma \in \mathcal{A}_{t, \nu}(\Sigma)} \mathbb{E}_{\mathbb{Q}}\left[h\left(S_{T}^{\gamma}\right)\right]
$$

Since the agent only knows the set $\Gamma$ where the volatility can assume its values, he associates the price of the claim at time 0 with the interval $\left[V^{-}\left(0, S_{0}\right), V^{+}\left(0, S_{0}\right)\right]$ of the real line, called the set of admissible prices. In fact, by definition of $V^{-}$and $V^{+}$, for all reference probability systems $\nu$ and $\gamma \in \mathcal{A}_{0, \nu}(\Sigma)$ we have

$$
V^{-}\left(0, S_{0}\right) \leq \mathbb{E}_{\mathbb{Q}}\left[h\left(S_{T}^{\gamma}\right)\right] \leq V^{+}\left(0, S_{0}\right)
$$

so that every possible arbitrage free price lies in the interval $\left[V^{-}\left(0, S_{0}\right), V^{+}\left(0, S_{0}\right)\right]$. It is a classical result of stochastic optimal control that, under our assumptions, $V^{+}$is the unique 
viscosity solution of Equation (3) (see Theorem 7 in Section 3). In an analogous way, $V^{-}$is the unique viscosity solution of Equation (3) provided we substitute the max operator with min.

We now present the main known result on this subject. We indicate with $C_{p}^{1,2}([0, T) \times$ $\left.\mathbb{R}_{+}^{n}\right)$ the space of functions that are continuous and with polynomial growth on $[0, T) \times \mathbb{R}_{+}^{n}$ together with their first derivative in $t$ and first and second derivatives in $s$. Theorem 5 below is stated (in a slightly different way) and proved in [20] (see also [1], [17] for previous similar results).

Theorem 5 If $V \in C_{p}^{1,2}\left([0, T) \times \mathbb{R}_{+}^{n}\right)$ is the solution of Equation (3) and $\Delta_{t}=D_{s} V\left(t, S_{t}\right)$, then $V\left(0, S_{0}\right)$ is the superreplication capital at time 0 and $\Delta$ is a Markov superstrategy.

This is a characterization theorem of the superstrategies. In fact, provided the value function $V \in C_{p}^{1,2}$, the theorem gives the minimal superstrategy, in the sense that there does not exist a superstrategy starting with an initial capital less than $V\left(0, S_{0}\right)$.

For completeness we give also the results for a substrategy.

Theorem 6 If $V \in C_{p}^{1,2}\left([0, T) \times \mathbb{R}_{+}^{n}\right)$ is the solution of Equation (3) (provided we substitute the max with min) and $\Delta_{t}=D_{s} V\left(t, S_{t}\right)$, then $V\left(0, S_{0}\right)$ is the subreplication capital at time 0 and $\Delta$ is a Markov substrategy.

We notice that the substrategy case is in some sense symmetric to that of the superstrategy. This has a simple mathematical explanation: in fact, if $V$ is the solution of the BSB equation with final condition $h$, then $-V$ is the solution of the BSB equation with min instead of max, and final condition $-h$. In other words, if $V$ defines a superstrategy for the claim defined by $h$, then $-V$ defines a substrategy for the claim defined by $-h$. This allows us to analyze only one of the two cases, and to have automatically results for the other. For this reason, in the rest of the paper we will analyze only the superstrategy case, and it will be implicit that analogous results will hold also for substrategies, unless otherwise stated.

The two theorems above require the existence of a $C^{1,2}$ solution of Equation (3) in order to have a Markov superstrategy. This smoothness requirement however is very strong (see e.g. $[11$, p. 162]). In the following section we will see what kind of regularity we can expect for the solution of Equation (3), while in Section 4 we see how to prove a weak version of Theorem 5 in the case when we do not know if the solution of Equation (3) is $C^{1,2}$.

\section{The BSB equation}

We collect here the main results on existence, uniqueness and regularity taken from [23].

The first step is to recall a general result for existence and uniqueness of viscosity solutions for the BSB equation (3) that always holds true in our case.

Theorem 7 Let $\Sigma$ be compact and $h \in C_{p}\left(\mathbb{R}_{+}^{n}\right)$. Then the value function $V^{+}$defined by Equation (6) is a viscosity solution of Equation (3) in $[0, T] \times \mathbb{R}_{+}^{n}$. Moreover, $V^{+}$is the unique viscosity solution having polynomial growth that satisfies the boundary condition $V(T, s)=h(s)$ for all $s \in \mathbb{R}_{+}^{n}$.

The second step is to prove smoothness of the viscosity solution. We have the following general result on the existence of the first weak derivative. 
Theorem 8 Let $\Sigma$ be compact and $h$ be locally Lipschitz continuous, and let $\|h\|_{\text {Lip }}$ be its Lipschitz constant. Moreover assume that for some $m \in \mathbb{N}$ we have ${ }^{1}$

$$
|h(s)| \leq M\left(1+|s|^{m}\right) \quad\left|D_{s} h(s)\right| \leq M\left(1+|s|^{m}\right) \quad \text { for a.e. } s \in \mathbb{R}_{+}^{n}
$$

Then, for every $p \in[1,+\infty]$ we have $D_{s} V \in L_{\mathrm{loc}}^{p}\left([0, T] \times \mathbb{R}_{+}^{n}\right)$ and for suitable $M>0, k \in \mathbb{N}$

$$
\left|D_{s} V(t, s)\right| \leq M\left(1+|s|^{k}\right)
$$

for almost every $(t, s) \in[0, T] \times \mathbb{R}_{+}^{n}$. Moreover, if $h$ is globally Lipschitz, then also $V$ is globally Lipschitz and, for a suitable $M>0$

$$
\left|D_{s} V(t, s)\right| \leq M\|h\|_{\text {Lip }}
$$

Now we present stronger regularity results involving the second derivative that come from known results on uniformly parabolic equations. The main references on regularity for uniformly parabolic equations are [5] and [24]. In order to use these results, we make a change of variable to transform the BSB equation (which is never uniformly parabolic) into another one. We put $y_{i}=\log s_{i}$. Then the BSB equation becomes:

$$
\begin{cases}\frac{\partial \tilde{V}}{\partial t}(t, y)+\frac{1}{2} \max _{\gamma \in \Sigma} \operatorname{tr}\left(\left(D_{s}^{2} \tilde{V}(t, y)-\operatorname{diag}\left(D_{s} \tilde{V}(t, y)\right)\right) \gamma \gamma^{*}\right)=0, & t \in[0, T), y \in \mathbb{R}^{n} \\ \tilde{V}(T, \cdot)=\tilde{h}(\cdot) & y \in \mathbb{R}^{n}\end{cases}
$$

where $\tilde{V}(t, y)=V\left(t,\left(e^{y_{i}}\right)_{i}\right)=V(t, s)$. In fact we can see that $V$ is a viscosity solution of Eq. (3) if and only if $\tilde{V}$ is a viscosity solution of Eq. (8) (see [2, p. 38-39] for change of variables in the theory of viscosity solutions). We notice that in the BSB equation (3) the domain is $\mathbb{R}_{n}^{+}$, while in this equation the domain is all $\mathbb{R}^{n}$. We now recall the following definition.

Definition 9 We say that Equation (8) is uniformly parabolic if there exists $M>m>0$ such that for all $\gamma \in \Sigma$ and $\xi \in \mathbb{R}^{n}$,

$$
\left.m\|\xi\|^{2} \leq\left\langle\gamma \gamma^{*} \xi, \xi\right\rangle\right\rangle \leq M\|\xi\|^{2}
$$

The above definition can be rewritten in this way:

$$
\exists M^{\prime}>m^{\prime}>0 \text { such that } \forall \gamma \in \Sigma, \xi \in \mathbb{R}^{n}, m^{\prime}\|\xi\| \leq\left\|\gamma^{*} \xi\right\| \leq M^{\prime}\|\xi\|
$$

This implies that if there exists a $\gamma \in \Sigma$ which does not have rank equal to $n$, then Equation (8) is not uniformly parabolic. In fact if we take $\xi \in \operatorname{ker} \gamma^{*}$, then $\left\|\gamma^{*} \xi\right\|=0$. We show that the converse is also true. To this purpose, we use the notation $\Sigma^{2}=\left\{\gamma \gamma^{*} \mid \gamma \in \Sigma\right\}$. Now we present a result, whose (easy) proof can be found in [20].

Lemma 10 If $\Sigma$ is closed and bounded in $M(n, d, \mathbb{R})$, then $E q$. (8) is uniformly parabolic if and only if $\Sigma^{2} \subseteq \operatorname{GL}(n, \mathbb{R})$, where $\operatorname{GL}(n, \mathbb{R})=\left\{\gamma \in \mathbb{R}^{n \times n} \mid \operatorname{det} \gamma \neq 0\right\}$.

\footnotetext{
${ }^{1}$ Recall that $D_{s} h$ is defined a.e. thanks to the Rademacher's Theorem.
} 
We notice that if $d<n$, then Eq. (8) can not be uniformly parabolic, because $\gamma$ (and consequently $\gamma \gamma^{*}$ ) has rank at most $d$ for $\gamma \in \Sigma$.

The above Lemma 10 allows us to state the following theorem. For $m \in \mathbb{N}, \alpha \in(0,1)$, we call $C_{p}^{m+\alpha}(\mathbb{R})$ the space of all functions that are of polynomial growth and Hölder continuous of order of $\frac{m+\alpha}{2}$ in time and differentiable of order $m$ in space with derivatives Hölder continuous of order $\alpha$.

Theorem 11 Let $\Sigma$ be compact, $h$ be locally Lipschitz continuous and let $h, D_{s} h$ have polynomial growth. If $\Sigma^{2} \subseteq \mathrm{GL}(n, \mathbb{R})$ then there exists $\alpha \in(0,1]$ such that the viscosity solution of the BSB equation (3) belongs to $C_{p}^{2+\alpha}\left([0, T) \times \mathbb{R}^{n}\right)$.

As recalled in Section 2 the above regularity results allow us to apply the Itô formula and then Theorem 5. The final conclusion is that if the set $\Sigma^{2}$ is a closed bounded set in $\operatorname{GL}(n, \mathbb{R})$, then there exists a Markov superstrategy, and it is given by the space derivative of the solution of Equation (3). Conversely, if this does not happen, then we cannot apply the Theorem 11 above, so we cannot say that $V \in C^{1,2}$ (nor apply Theorem 5 ) without proving new regularity results that are unknown at this stage. However this case is interesting from the financial point of view as the following example, taken from [20], shows.

Example 12 Consider the case of 2 assets, each one with known volatility but with unknown mutual correlation, which we assume to follow a stochastic process. Then

$$
\Sigma=\left\{\gamma \in M(n, n, \mathbb{R}) \mid \gamma=\left(\begin{array}{cc}
\sigma_{1} & 0 \\
\rho \sigma_{2} & \sqrt{1-\rho^{2}} \sigma_{2}
\end{array}\right), \quad \rho \in[-1,1]\right\}
$$

As indicated in [20], this corresponds to the case when the volatilities $\sigma_{1}, \sigma_{2}$ of the two assets $S_{1}$ and $S_{2}$ are known, but the correlation is not. In particular we allow the Brownian motion driving $S_{1}$ and $S_{2}$ to be in all the states between perfectly positively correlated $(\rho=1)$ and perfectly negatively correlated $(\rho=-1)$, passing through a null correlation $(\rho=0)$. We can see that for $\rho= \pm 1$ we have that $\gamma \gamma^{*} \notin \mathrm{GL}(n, \mathbb{R})$ and the BSB equation (3) is not uniformly parabolic. This is a typical situation arising when we want to price options on two correlated assets. In this case typically one has a good estimate for $\sigma_{1}$ and $\sigma_{2}$ but not for the correlation $\rho$ which is more difficult to estimate.

Remark 13 The BSB equation (3) can also be formulated by adding suitable boundary conditions when $s=0$ and $s \rightarrow+\infty$ (on this see [7, p. 47]). We did not put these conditions here since they are automatically satisfied in our case by the viscosity solutions (see e.g. $[3,11,12])$

\section{The superstrategy}

In this section we state and prove a weaker version of Theorem 5 in the case when uniform parabolicity does not hold. For the sake of simplicity, we make the assumption that $d=n$, but the result remains true (with heavier notation) also for $d>n$. In particular we will prove that also in the non-uniformly parabolic case the quantities $V\left(0, S_{0}\right)$ and $\Delta$ defined in Section 2 are respectively the superreplication capital at time 0 and a Markov superstrategy when the final payoff $h$ is a convex (or semiconvex) function of the assets and the law of the price $S_{t}$ is absolutely continuous with respect to the Lebesgue measure for all $t \in(0, T]$. This case does not cover all the possible payoffs (for example, it does not cover call-spreads), 
but indeed many of the multiasset European options (call options on the maximum, call and put options on the mean, exchange options, etc.) are covered.

We observe that for the analogous results for a substrategy one needs to assume that $h$ is concave, not convex and so the symmetry between sub- and superstrategies breaks here. So, if we want to get an existence result for substrategies when $h$ is convex we need the additional assumption that the set $\Sigma$ be convex (see Remark 18 below).

We start by recalling our assumptions.

Hypothesis 14 (i) The payoff $h$ is convex and Lipschitz continuous ( $\|h\|_{\text {Lip }}$ will denote its Lipschitz constant).

(ii) For every $t>0$ the law of the random variable $S_{t}^{\sigma}$ has a density with respect to the Lebesgue measure which is absolutely continuous.

From these assumptions, we can prove the following theorem, which is the main result of this paper. It essentially says that, even when the coefficients of the BSB equation give poor regularity, it is still possible to characterize the superreplication capital and to build a Markov superstrategy via the solution of the BSB equation, provided we have a suitable terminal condition $h$ and regularity of the marginal laws of the price process.

Theorem 15 If Hypothesis 14 holds and $V$ denotes the unique viscosity solution of Eq. (3), then $V\left(0, S_{0}\right)$ is the superreplication capital at time $0, \Delta_{t}=D_{s} V\left(t, S_{t}\right)$ is $\mathbb{Q}$-a.s. well defined and is a Markov superstrategy.

Remark 16 We recall that all the results given below continue to hold true if the function $h$ is only semi-convex, of polynomial growth, and locally Lipschitz. Since this extension has no great interest in financial examples and would complicate the readability of the proofs, we do not treat it.

In the proof of this theorem, we will use several intermediate results. First of all we approximate the solution $V$ of the non-uniformly parabolic BSB equation (3) with a sequence $\left(V_{\varepsilon_{n}}\right)_{n}$, where $V_{\varepsilon}$ is the solutions of a uniformly parabolic BSB equation, as follows. For each $\varepsilon \in[0,1)$, we consider the equation

$$
\left\{\begin{array}{l}
\frac{\partial V}{\partial t}(t, s)+\frac{1}{2} \max _{\gamma \in \Sigma_{\varepsilon}} \operatorname{tr}\left(D^{2} V(t, s)(\bar{s} \gamma)(\bar{s} \gamma)^{*}\right)=0 \\
V(T, \cdot)=h(\cdot)
\end{array}\right.
$$

where $\Sigma_{\varepsilon}=\Sigma+\varepsilon I$. This corresponds to the control problem having as controlled process

$$
d S_{t}^{\gamma, \varepsilon}=S_{t}^{\gamma, \varepsilon} \gamma_{t} d W_{t}+S_{t}^{\gamma, \varepsilon}(\varepsilon I) d \bar{W}_{t}
$$

with $\gamma \in \mathcal{A}(\Sigma)$, and as value function

$$
V_{\varepsilon}(t, s)=\sup _{\gamma \in \mathcal{A}(\Sigma)} \mathbb{E}\left[h\left(S_{T}^{\gamma, \varepsilon}\right) \mid S_{t}^{\gamma, \varepsilon}=s\right]
$$

where $\bar{W}_{t}$ is an $n$-dimensional Brownian motion independent of $\mathcal{F}_{t}$. To be precise, here we consider a reference probability system $\mu=\left(\Omega \times \Omega^{\prime},\left(\mathcal{F}_{s}^{\prime}\right)_{s \in[t, T]}, \mathbb{Q} \otimes \mathbb{Q}^{\prime},(W, \bar{W})\right)$, where $\Omega^{\prime}$ is the canonical Wiener space of $\bar{W}, \mathcal{F}_{s}^{\prime}=\overline{\mathcal{F}_{s} \vee \sigma\left(\bar{W}_{u}, u \leq s\right)}$ and $\mathbb{Q}^{\prime}$ is the Wiener 
measure of $\bar{W}$ (see [11] for details). To avoid this heavy notation, we will indicate this enlarged reference probability system as usual by $\nu=\left(\Omega,\left(\mathcal{F}_{s}\right)_{s \in[t, T]}, \mathbb{Q},(W, \bar{W})\right)$. We have that $S_{t}^{\gamma, \varepsilon}=S_{t}^{\gamma} \mathcal{E}_{t}(\varepsilon \bar{W})$, where we indicate with $\mathcal{E}_{t}(\varepsilon \bar{W})=\exp \left(\varepsilon \bar{W}_{t}-1 / 2 \varepsilon^{2} t\right)$ the DoleansDale exponential of the process $\varepsilon \bar{W}$ calculated in $t$. Note that these control problems are completely artificial and are used only to get properties in the limit as $\varepsilon \rightarrow 0$ (for $\varepsilon=0$ we obtain the original control problem).

Lemma 17 For all $\varepsilon \geq 0$, the function $V_{\varepsilon}$ is the unique viscosity solution of Equation (3) in the class $C_{p}\left([0, T] \times \mathbb{R}_{+}^{n}\right)$, and it is convex. Moreover, for all $\varepsilon>0, V_{\varepsilon}$ is of class $C^{1,2}$ with Hölder continuous derivatives.

Proof. Existence, uniqueness and regularity follow from Theorems 7 and 11 of Section 3. To prove convexity we use substantially that the supremum of convex functions is convex and the linearity of the equation for the price process $S_{t}^{\gamma, \varepsilon}$. In fact, denoting by $S_{u}^{\gamma, \varepsilon}(t, s)$ the solution of Equation (10) with initial condition $S_{t}^{\gamma, \varepsilon}=s$, we have that for $\lambda \in[0,1]$ and $s_{1}, s_{2} \in \mathbb{R}^{n}$,

$$
S_{u}^{\gamma, \varepsilon}\left(t, \lambda s_{1}+(1-\lambda) s_{2}\right)=\lambda S_{u}^{\gamma, \varepsilon}\left(t, s_{1}\right)+(1-\lambda) S_{u}^{\gamma, \varepsilon}\left(t, s_{2}\right)
$$

Then by definition of $V_{\varepsilon}$ and by using the linearity of the equation for the price process $S_{t}^{\gamma, \varepsilon}$

$$
\begin{aligned}
V_{\varepsilon}\left(t, \lambda s_{1}+(1-\lambda) s_{2}\right) & =\sup _{\gamma \in \Sigma} \mathbb{E}\left[h\left(S_{T}^{\gamma, \varepsilon}\left(t, \lambda s_{1}+(1-\lambda) s_{2}\right)\right]=\right. \\
& =\sup _{\gamma \in \Sigma} \mathbb{E}\left[h\left(\lambda S_{T}^{\gamma, \varepsilon}\left(t, s_{1}\right)+(1-\lambda) S_{T}^{\gamma, \varepsilon}\left(t, s_{2}\right)\right)\right] \leq \\
& \leq \sup _{\gamma \in \Sigma} \mathbb{E}\left[\lambda h\left(S_{T}^{\gamma, \varepsilon}\left(t, s_{1}\right)\right)+(1-\lambda) h\left(S_{T}^{\gamma, \varepsilon}\left(t, s_{2}\right)\right)\right] \leq \\
& \leq \sup _{\gamma \in \Sigma} \mathbb{E}\left[h\left(S_{T}^{\gamma, \varepsilon}\left(t, s_{1}\right)\right)\right]+(1-\lambda) \sup _{\gamma \in \Sigma} \mathbb{E}\left[h\left(S_{T}^{\gamma, \varepsilon}\left(t, s_{2}\right)\right)\right]
\end{aligned}
$$

which gives the claim.

Remark 18 We observe that in the case of substrategy, to get convexity of $V_{\varepsilon}$ we would need the further assumption that the set $\Sigma$ is convex. In fact in this case the last step would not work since we have inf instead of sup. Convexity of $\Sigma$ ensures that, for every given admissible strategies $\gamma_{1}$ and $\gamma_{2}$ we can find an admissible $\gamma_{\lambda}$ such that

$$
S_{T}^{\gamma_{\lambda}, \varepsilon}\left(t, \lambda s_{1}+(1-\lambda) s_{2}\right)=\lambda S_{T}^{\gamma_{1}, \varepsilon}\left(t, s_{1}\right)+(1-\lambda) S_{T}^{\gamma_{2}, \varepsilon}\left(t, s_{2}\right)
$$

This allows us to repeat the final step of the proof above. Then, under the assumption of convexity of $\Sigma$, all the results of this section can be repeated for the substrategy case.

Now we show that the functions $V_{\varepsilon}$ converge pointwise to $V$ and that there exists a sequence $\left(V_{\varepsilon_{n}}\right)_{n}$ such that $\left(D_{s} V_{\varepsilon_{n}}\right)_{n}$ converges in $L^{p}$ to $D_{s} V$. This follows from an estimate on the weak first derivatives of $V_{\varepsilon}, \varepsilon>0$, and $V$, based on estimates on the function $h$, and from the fact that the $V_{\varepsilon}, \varepsilon>0$, and $V$ are convex.

Proposition 19 Let $V_{\varepsilon}$ be the viscosity solution of $E q$. (9) and let $V$ be the viscosity solution of Eq. (3). Then $V_{\varepsilon} \rightarrow V$ pointwise for $\varepsilon \rightarrow 0$, and there exists a subsequence $\left(\varepsilon_{k}\right)_{k}$ such that $D_{s} V_{\varepsilon_{k}}$ converges to $D_{s} V$ in $L_{\text {loc }}^{p}\left((0, T) \times \mathbb{R}_{+}^{n}\right)$ and Lebesgue-almost surely in $(0, T) \times \mathbb{R}_{+}^{n}$. 
In the proof, we will use a lemma, due to P. L. Lions, which we present in a slightly different formulation.

Lemma 20 Let $\left(u_{n}\right)_{n>0}$ be a bounded family of concave functions in $L^{\infty}([0, T] \times B)$, with $B$ a bounded set in $\mathbb{R}_{+}^{n}$, such that $\left(D_{s} u_{n}\right)_{n}$ is bounded in $L^{\infty}\left([0, T] \times B ; \mathbb{R}^{n}\right)$. Then $\left(D_{s} u_{n}\right)_{n}$ is relatively compact in $L^{p}([0, T] \times B)$ for all $p \in[1, \infty)$.

Proof. The proof is the same as in [16, p. 209], by noticing that in that proof the time derivatives $D_{t} u_{n}$ plays no role.

Now we prove Proposition 19.

Proof. First we prove that $V_{\varepsilon} \rightarrow V$ pointwise. We show that the difference $V_{\varepsilon}(t, s)-V(t, s)$ goes to 0 as $\varepsilon \rightarrow 0$. We will show only one inequality as the other one can be obtained with exactly the same procedure. Taking $\gamma_{\delta}$ a $\delta$-suboptimal control for $V_{\varepsilon}(t, s)$ we have

$$
\begin{aligned}
V_{\varepsilon}(t, s)-V(t, s) & =\sup _{\gamma \in \Sigma} \mathbb{E}\left[h\left(S_{T}^{\gamma, \varepsilon}(t, s)\right]-\sup _{\gamma \in \Sigma} \mathbb{E}\left[h\left(S_{T}^{\gamma}(t, s)\right] \leq\right.\right. \\
& \leq \delta+\mathbb{E}\left[h\left(S_{T}^{\gamma \delta, \varepsilon}(t, s)\right]-\mathbb{E}\left[h\left(S_{T}^{\gamma \delta}(t, s)\right] \leq\right.\right. \\
& \leq \delta+\mathbb{E}\left[\|h\|_{\operatorname{Lip}}\left|S_{T}^{\gamma_{\delta}}(t, s)(\mathcal{E}(\varepsilon \bar{W})-1)\right|\right] \leq \\
& \leq \delta+\|h\|_{\operatorname{Lip}} \mathbb{E}\left[\left|S_{T}^{\gamma_{\delta}}(t, s)\right|^{2}\right]^{1 / 2} \cdot \mathbb{E}\left[\left|\mathcal{E}_{T}(\varepsilon \bar{W})-1\right|^{2}\right]^{1 / 2}
\end{aligned}
$$

Since we have the estimate (see $[11,14])$

$$
\mathbb{E}\left[\left|S_{T}^{\gamma_{\delta}}(t, s)\right|^{2}\right] \leq B_{2}\left(1+|s|^{2}\right)
$$

which is independent of $\delta$ and $\varepsilon$, the claim follows by the mean square convergence to 0 of the random variable $\mathcal{E}(\varepsilon \bar{W})-1$ as $\varepsilon$ tends to 0 . Note that the convergence that we obtain is uniform on bounded sets of $[0, T] \times \mathbb{R}_{+}^{n}$.

Now we prove that $D_{s} V_{\varepsilon} \stackrel{L_{\text {loc }}^{p}}{\longrightarrow} D_{s} V$ : we have that $\left\|D_{s} V_{\varepsilon}(t, s)\right\|_{\infty} \leq\|h\|_{\text {Lip }}$ uniformly in $\varepsilon$. If we fix a compact set $B$ in $\mathbb{R}_{+}^{n}$ and $p \in[1,+\infty)$, then the sequence $\left(D_{s} V_{1 / n}\right)_{n}$ is bounded in $L^{\infty}([0, T] \times B)$, and the functions $-V_{1 / n}$ are concave. Then, by Lemma $(20),\left(D_{s} V_{1 / n}\right)_{n}$ is relatively compact in $L^{p}([0, T] \times B)$, so there is a subsequence $\left(D_{s} V_{\varepsilon_{k}}\right)_{k}$ and a $f \in L^{p}(B)$ such that $-D_{s} V_{\varepsilon_{k}} \rightarrow f$ in $L^{p}(B)$. Moreover, for all $g \in C_{0}^{\infty}(B)$ we have that

$$
\left\langle D_{s} V_{\varepsilon_{k}}, g\right\rangle_{L^{p}(B)}=-\left\langle V_{\varepsilon_{k}}, D_{s} g\right\rangle_{L^{p}(B)} \rightarrow-\left\langle C, D_{s} g\right\rangle_{L^{p}(B)}=\left\langle D_{s} C, g\right\rangle_{L^{p}(B)}
$$

Since $C_{0}^{\infty}(B)$ is dense in $L^{p}(B)$, we obtain that $D_{s} V_{\varepsilon_{k}} \stackrel{L_{\text {loc }}^{p}}{\longrightarrow} D_{s} V$. This implies that there exists another subsequence, which we still call $\left(\varepsilon_{k}\right)_{k}$ such that $D_{s} V_{\varepsilon_{k}} \longrightarrow D_{s} V$ a.s. in $(0, T) \times \mathbb{R}^{n}$. This completes the proof.

Now we use these convergence results in order to prove that the superreplication price $V\left(0, S_{0}\right)$ is the limit of the quantities $V_{\varepsilon_{k}}\left(0, S_{0}\right)$, and that the superhedging portfolio $\Pi_{t}=$ $D_{s} V\left(t, S_{t}\right)$ is the pointwise limit of portfolios $\Pi^{k}$ defined via the functions $V_{\varepsilon_{k}}$. From now on, for brevity we call $V_{k}=V_{\varepsilon_{k}}$ and recalling that the true (but unknown) volatility is denoted by $\sigma$ we will write $S_{t}^{k}$ in place of $S_{t}^{\sigma, \varepsilon_{k}}$. We also write $V_{t}^{k}$ for $V_{\varepsilon_{k}}\left(t, S_{t}^{k}\right)$ and use $\Pi^{k}$ to denote the corresponding superreplicating portfolio, defined by $\Pi_{t}^{k}=D_{s} V_{k}\left(t, S_{t}^{k}\right)$.

We present now three approximation results that will lead to the proof of the main theorem. 
Proposition 21 The process $S^{k}$ tends to $S$ uniformly in $t \in[0, T], \mathbb{Q}$-a.s.

Proof. Since $S^{k}-S$ is a martingale for all $k$, by Doob's inequality we have that

$$
\mathbb{E}\left[\sup _{t \in[0, T]}\left|S_{t}^{k}-S_{t}\right|^{2}\right] \leq \sup _{t \in[0, T]} \mathbb{E}\left[\left|S_{T}^{k}-S_{T}\right|^{2}\right]
$$

Since $S_{T}^{k} \rightarrow S_{T} \mathbb{Q}$-a.s. and $\left|S_{T}^{k}-S_{T}\right|$ is dominated by $S_{T}\left(1+\mathcal{E}_{T}(\bar{W})\right) \in L^{2}$, by Lebesgue's Dominated Convergence theorem the conclusion follows.

Proposition 22 If Hypothesis 14 holds then $V^{k} \rightarrow V$, uniformly in $t \in[0, T], \mathbb{Q}$-a.s. on a suitable subsequence.

Proof. Recall that, thanks to the proof of Proposition 19 the functions $\left(V_{k}\right)_{k}$ converge to $V$ uniformly on bounded subsets of $[0, T] \times \mathbb{R}_{+}^{n}$. Then, for every $R>0$ there exists a modulus $\omega_{R}$ such that

$$
\sup _{t \in[0, T],|s| \leq R}\left|V_{k}(t, s)-V(t, s)\right| \leq \omega_{R}(1 / k)
$$

so that, for suitable $M_{1}>0$,

$$
\begin{aligned}
& \mathbb{E}\left[\sup _{t \in[0, T]}\left|V_{k}\left(t, S_{t}^{k}\right)-V\left(t, S_{t}^{k}\right)\right|\right] \leq \\
& \leq \omega_{R}(1 / k)+\mathbb{E}\left[\sup _{t \in[0, T]}\left(\left|V_{k}\left(t, S_{t}^{k}\right)-V\left(t, S_{t}^{k}\right)\right| \mathbf{1}_{S_{t}^{k}>R}\right)\right] \leq \\
& \leq \omega_{R}(1 / k)+\mathbb{E}\left[\left(\sup _{t \in[0, T]}\left|V_{k}\left(t, S_{t}^{k}\right)-V\left(t, S_{t}^{k}\right)\right|\right) \mathbf{1}_{\left\|S^{k}\right\|_{\infty}>R}\right] \leq \\
& \leq \omega_{R}(1 / k)+\mathbb{E}\left[\sup _{t \in[0, T]}\left|V_{k}\left(t, S_{t}^{k}\right)-V\left(t, S_{t}^{k}\right)\right|^{2}\right]^{1 / 2} \mathbb{Q}\left\{\left\|S^{k}\right\|_{\infty}>R\right\}^{1 / 2} \leq \\
& \leq \omega_{R}(1 / k)+\frac{M_{1}}{R} \mathbb{E}\left[\left(1+\left\|S^{k}\right\|_{\infty}\right)^{2}\right]^{1 / 2} \mathbb{E}\left[\left\|S^{k}\right\|_{\infty}^{2}\right]^{1 / 2}
\end{aligned}
$$

Using this estimate, and setting $V_{t}=V\left(t, S_{t}^{\sigma}\right)$, we find that, for every $R>0$,

$$
\begin{aligned}
& \mathbb{E}\left[\sup _{t \in[0, T]}\left|V_{t}^{k}-V_{t}\right|\right] \leq \\
& \leq \mathbb{E}\left[\sup _{t \in[0, T]}\left|V_{k}\left(t, S_{t}^{k}\right)-V\left(t, S_{t}^{k}\right)\right|\right]+\mathbb{E}\left[\sup _{t \in[0, T]}\left|V\left(t, S_{t}^{k}\right)-V\left(t, S_{t}^{\sigma}\right)\right|\right] \\
& \leq \omega_{R}(1 / k)+M_{2} \frac{M_{1}}{R} \mathbb{E}\left[\left(1+\left\|S^{k}\right\|_{\infty}\right)^{2}\right]^{1 / 2} \mathbb{E}\left[\left\|S^{k}\right\|_{\infty}^{2}\right]^{1 / 2}+M_{3} \mathbb{E}\left[\left\|S^{\sigma} \cdot\left(\mathcal{E} .\left(\varepsilon_{k} \bar{W}\right)-1\right)\right\|_{\infty}\right]
\end{aligned}
$$

for suitable $M_{2}, M_{3}>0$. Taking $R$ sufficiently large and then letting $k \rightarrow \infty$ we get

$$
\lim _{k \rightarrow+\infty} \mathbb{E}\left[\left\|V_{t}^{k}-V_{t}\right\|_{\infty}\right]=0 .
$$


This gives the a.s. uniform convergence on a suitable subsequence.

For the third result, we define $\bar{\Pi}_{t}^{k}=\int_{0}^{t}\left\langle D_{s} V_{k}\left(u, S_{u}^{k}\right), d S_{u}^{k}\right\rangle$ and $\bar{\Pi}_{t}=$ $\int_{0}^{t}\left\langle D_{s} V\left(u, S_{u}^{\sigma}\right), d S_{u}^{\sigma}\right\rangle$.

Proposition 23 If Hypothesis 14 holds then $\bar{\Pi}^{k} \rightarrow \bar{\Pi}$ a.s., uniformly in $t \in[0, T]$ on a suitable subsequence.

Proof. Without loss of generality we can renumber the sequence $\bar{\Pi}^{k}$ on $k$ on the basis of the limiting subsequence of the previous proposition. We then have

$$
\begin{aligned}
\bar{\Pi}_{t}^{k}-\bar{\Pi}_{t}= & \int_{0}^{t}\left\langle D_{s} V_{k}\left(u, S_{u}^{k}\right)-D_{s} V_{k}\left(u, S_{u}^{\sigma}\right), \bar{S}_{u}^{k} \sigma_{u} d W_{u}\right\rangle+ \\
& +\int_{0}^{t}\left\langle D_{s} V_{k}\left(u, S_{u}^{\sigma}\right)-D_{s} V\left(u, S_{u}^{\sigma}\right), \bar{S}_{u}^{k} \sigma_{u} d W_{u}\right\rangle+ \\
& +\int_{0}^{t}\left\langle D_{s} V\left(u, S_{u}^{\sigma}\right),\left[\bar{S}_{u}^{k}-\bar{S}_{u}^{\sigma}\right] \sigma_{u} d W_{u}\right\rangle+\int_{0}^{t}\left\langle D_{s} V_{k}\left(u, S_{u}^{k}\right), \bar{S}_{u}^{k} \varepsilon_{k} d \bar{W}_{u}\right\rangle
\end{aligned}
$$

We now denote by $(i),(i i),(i i i)$ and $(i v)$ the terms on the right hand side, and estimate their mean square. For the first one, setting $\|\Sigma\|=\sup _{\sigma \in \Sigma}\|\sigma\|_{M(n, n ; \mathbb{R})}$ we have:

$$
\begin{aligned}
\mathbb{E}\left[|(i)|^{2}\right] & \leq\|\Sigma\|^{2} \mathbb{E} \int_{0}^{t}\left\|\bar{S}_{u}^{k}\right\|^{2}\left\|D_{s} V_{k}\left(u, S_{u}^{k}\right)-D_{s} V_{k}\left(u, S_{u}^{\sigma}\right)\right\|^{2} d u \leq \\
& \leq\|\Sigma\|^{2}\left[\mathbb{E} \int_{0}^{t}\left\|\bar{S}_{u}^{k}\right\|^{4} d u\right]^{1 / 2}\left[\mathbb{E} \int_{0}^{t}\left\|D_{s} V_{k}\left(u, S_{u}^{k}\right)-D_{s} V_{k}\left(u, S_{u}^{\sigma}\right)\right\|^{4} d u\right]^{1 / 2}
\end{aligned}
$$

Now we use the boundedness of $\mathbb{E}\left[\left\|\bar{S}_{u}^{k}\right\|^{4}\right]$ to get

$$
\mathbb{E}\left[|(i)|^{2}\right] \leq M\left[\mathbb{E} \int_{0}^{t}\left\|D_{s} V_{k}\left(u, S_{u}^{k}\right)-D_{s} V_{k}\left(u, S_{u}^{\sigma}\right)\right\|^{4} d u\right]^{1 / 2}
$$

for suitable $M>0$. At this point we use Hypothesis 14-(ii) and the independence of $\mathcal{F}$ and $\bar{W}$. In fact, taking conditional expectation:

$$
\begin{aligned}
\mathbb{E} & \int_{0}^{t}\left\|D_{s} V_{k}\left(u, S_{u}^{k}\right)-D_{s} V_{k}\left(u, S_{u}^{\sigma}\right)\right\|^{4} d u= \\
& =\int_{0}^{t} \mathbb{E}\left[\mathbb{E}\left[\left\|D_{s} V_{k}\left(u, S_{u}^{k}\right)-D_{s} V_{k}\left(u, S_{u}^{\sigma}\right)\right\|^{4} \mid \bar{W}_{u}\right]\right] d u \\
& =\int_{0}^{t} \mathbb{E}\left[\int_{\mathbb{R}_{+}^{n}}\left\|D_{s} V_{k}\left(u, s \mathcal{E}\left(\varepsilon_{k} \bar{W}_{u}\right)\right)-D_{s} V_{k}(u, s)\right\|^{4} \rho_{S_{u}^{\sigma}}(s) d s\right] d u
\end{aligned}
$$

Now we recall that the sequence $\left(D_{s} V_{k}\right)_{k \in \mathbb{N}}$ converges a.e. in $[0, T] \times \mathbb{R}_{+}^{n}$ and is bounded, so by the dominated convergence theorem it is compact in the space $L^{p}\left([0, T] \times \mathbb{R}_{+}^{n}\right)$ with weight $\rho_{S_{u}^{\sigma}}(s)$. It follows that, by the change of variable $s_{i}=e^{z_{i}}$ also the sequence $\left(D_{s} V_{k}(\cdot, e)\right)_{k \in \mathbb{N}}$ is compact in the space $L^{p}\left([0, T] \times \mathbb{R}^{n}\right)$ with weight $\rho_{S_{u}^{\sigma}}\left(e^{z}\right) e^{z}$. We can then apply the Fréchet-Kolmogorov Theorem (see [26, p. 275], where only the case of $L^{p}(\mathbb{R})$ without weight 
is considered: however the proof can be easily extended to our weighted spaces) to our case getting

$$
\begin{array}{rl}
\int_{0}^{t} & \mathbb{E}\left[\int_{\mathbb{R}_{+}^{n}}\left\|D_{s} V_{k}\left(u, s \mathcal{E}\left(\varepsilon_{k} \bar{W}_{u}\right)\right)-D_{s} V_{k}(u, s)\right\|^{4} \rho_{S_{u}^{\sigma}}(s) d s\right] d u= \\
\quad= & \mathbb{E} \int_{0}^{t}\left[\int_{\mathbb{R}^{n}} \| D_{s} V_{k}\left(u, e^{z+\varepsilon_{k} \bar{W}_{u}-\varepsilon_{k}^{2} u / 2}-D_{s} V_{k}\left(u, e^{z}\right) \|^{4} \rho_{S_{u}^{\sigma}}\left(e^{z}\right) e^{z} d z\right] d u \leq\right. \\
\quad \leq \mathbb{E} \int_{0}^{t} \omega\left(\left|\varepsilon_{k} \bar{W}_{u}-\varepsilon_{k}^{2} u / 2\right|\right) d u \stackrel{k \rightarrow+\infty}{\longrightarrow} 0
\end{array}
$$

(here $\omega$ denotes a modulus). For the second term we have

$$
\begin{aligned}
\mathbb{E}\left[|(i i)|^{2}\right] & \leq\|\Sigma\|^{2} \mathbb{E} \int_{0}^{t}\left\|\bar{S}_{u}^{\sigma}\right\|^{2}\left\|D_{s} V_{k}\left(u, S_{u}^{\sigma}\right)-D_{s} V\left(u, S_{u}^{\sigma}\right)\right\|^{2} d u \leq \\
& \leq\|\Sigma\|^{2}\left[\mathbb{E} \int_{0}^{t}\left\|\bar{S}_{u}^{\sigma}\right\|^{4} d s\right]^{1 / 2}\left[\mathbb{E} \int_{0}^{t}\left\|D_{s} V_{k}\left(u, S_{u}^{\sigma}\right)-D_{s} V\left(u, S_{u}^{\sigma}\right)\right\|^{4} d u\right]^{1 / 2} \leq \\
& \leq M\left[\mathbb{E} \int_{0}^{t}\left\|D_{s} V_{k}\left(u, S_{u}^{\sigma}\right)-D_{s} V\left(u, S_{u}^{\sigma}\right)\right\|^{4} d u\right]^{1 / 2}
\end{aligned}
$$

for suitable $M>0$. Then, using again Hypothesis 14-(ii) we get

$$
\mathbb{E} \int_{0}^{t}\left\|D_{s} V_{k}\left(u, S_{u}^{\sigma}\right)-D_{s} V\left(u, S_{u}^{\sigma}\right)\right\|^{4} d u=\int_{0}^{t} \int_{\mathbb{R}_{+}^{n}}\left\|D_{s} V_{k}(u, s)-D_{s} V(u, s)\right\|^{4} \rho_{S_{u}^{\sigma}}(s) d s d u
$$

which goes to 0 as $k \rightarrow+\infty$ thanks to the dominated convergence theorem.

Finally we have, for the third term, using Theorem 8:

$$
\begin{aligned}
\mathbb{E}\left[|(i i i)|^{2}\right] & =\mathbb{E}\left[\int_{0}^{t}\left\|D_{s} V\left(u, S_{u}^{\sigma}\right)\right\|^{2}\left|S_{u}^{k}-S_{u}^{\sigma}\right|^{2}\left\|\sigma_{u}\right\|^{2} d u\right] \leq \\
& \leq\|\Sigma\|^{2} M\|h\|_{\text {Lip }}^{2} \int_{0}^{t} \mathbb{E}\left[\left|S_{u}^{\sigma}\right|^{2}\left(1-\mathcal{E}_{u}\left(\varepsilon_{k} \bar{W}\right)\right)^{2}\right] d u \leq \\
& \leq M\|\Sigma\|^{2}\|h\|_{\text {Lip }}^{2} T\left(\mathbb{E}\left[\left\|S_{u}\right\|_{\infty}^{4}\right]\right)^{1 / 2}\left(\mathbb{E}\left[\left\|1-\mathcal{E} \cdot\left(\varepsilon_{k} \bar{W}\right)\right\|_{\infty}^{4}\right]\right)^{1 / 2}
\end{aligned}
$$

The last term tends to 0 as $k \rightarrow \infty$, so $\mathbb{E}\left[|(i i i)|^{2}\right] \rightarrow 0$. Similarly for the fourth term we have:

$$
\begin{aligned}
\mathbb{E}\left[|(i v)|^{2}\right] & =\varepsilon_{k}^{2} \mathbb{E}\left[\int_{0}^{t}\left\|D_{s} V_{k}\left(u, S_{u}^{k}\right)\right\|^{2}\left\|S_{u}^{k}\right\|^{2} d u\right] \leq \\
& \leq \varepsilon_{k}^{2} M T\|h\|_{\text {Lip }}^{2} \mathbb{E}\left[\left\|S_{.}^{k}\right\|_{\infty}^{2}\right] \leq \varepsilon_{k}^{2} M T\|h\|_{\text {Lip }}^{2} B_{2}\left(1+|s|^{2}\right)
\end{aligned}
$$

so $\mathbb{E}\left[|(i v)|^{2}\right] \rightarrow 0$ as $k \rightarrow \infty$.

To conclude we have obtained that for all $t \geq 0$

$$
\lim _{k \rightarrow \infty} \mathbb{E}\left[\left|\bar{\Pi}_{t}^{k}-\bar{\Pi}_{t}\right|^{2}\right]=0
$$

for all $t \in[0, T]$. Since $\bar{\Pi}^{k}-\bar{\Pi}$ is a martingale, we also have, thanks to Doob's inequality, that

$$
\lim _{k \rightarrow \infty} \mathbb{E}\left[\sup _{t \in[0, T]}\left|\bar{\Pi}_{t}^{k}-\bar{\Pi}_{t}\right|^{2}\right]=0
$$


This means that, on a suitable subsequence, $\lim _{k \rightarrow \infty} \bar{\Pi}_{t}^{k}=\bar{\Pi}_{t}$ almost surely, and the limit is uniform in $t$.

Now we are able to prove our main result.

Proof. First we prove that the superreplication capital $V^{+}$cannot be less than the solution $V$ of the BSB equation. Suppose by way of contradiction that $V^{+}\left(0, S_{0}\right)<V\left(0, S_{0}\right)$. Since $V$ is equal to the value function (6), this means that for all $\varepsilon \in\left(0, V\left(0, S_{0}\right)-V^{+}\left(0, S_{0}\right)\right)$ there exists an admissible volatility $\gamma \in \mathcal{A}(\Sigma)$ and a forward measure $\mathbb{Q}^{\prime}$ such that $V^{+}\left(0, S_{0}\right)+$ $\varepsilon<\mathbb{E}_{\mathbb{Q}^{\prime}}\left[h\left(S_{T}^{\gamma}\right)\right]$. By definition of superreplication capital, there exists $\Delta$ admissible such that $V^{+}\left(0, S_{0}\right)+\varepsilon+\int_{0}^{T}\left\langle\Delta_{t}, d S_{t}^{\gamma}\right\rangle \geq h\left(S_{T}^{\gamma}\right)$. Since $\Pi$ is a supermartingale, by taking the expectation with respect to $\mathbb{Q}^{\prime}$ we obtain

$$
V^{+}\left(0, S_{0}\right)+\varepsilon \geq \mathbb{E}_{\mathbb{Q}^{\prime}}\left[V^{+}\left(0, S_{0}\right)+\varepsilon+\int_{0}^{T}\left\langle\Delta_{t}, d S_{t}^{\gamma}\right\rangle\right] \geq \mathbb{E}_{\mathbb{Q}^{\prime}}\left[h\left(S_{T}^{\gamma}\right)\right]
$$

which, combined with the initial assumption, gives a contradiction.

Now we show that, starting with the initial capital $V\left(0, S_{0}\right)$, it is possible to build a Markov superstrategy. This will automatically imply the converse inequality $V^{+}\left(0, S_{0}\right) \leq V\left(0, S_{0}\right)$. Since $V \in W_{\text {loc }}^{1, p}$ and for all $t \in[0, T], S_{t}$ has a law which is absolutely continuous with respect to the Lebesgue measure, then $\Delta_{t}=D_{s} V\left(t, S_{t}\right)$ is a.s. well defined. By definition of superreplication capital, thanks to Theorem 5 for all $\varepsilon>0$ we have $\mathbb{Q}$-a.s. that $V_{0}^{k}+\varepsilon+\Pi_{T}^{k} \geq h\left(S_{T}^{k}\right)$ for all $k \in \mathbb{N}$. Thanks to Propositions 21, 22 and 23 , by passing to the limit on a suitable subsequence we have $\mathbb{Q}$-a.s. that $V_{0}+\varepsilon+\Pi_{T} \geq h\left(S_{T}\right)$. Since this result holds for all $\varepsilon>0$, it follows that $V_{0}=V\left(0, S_{0}\right) \geq V^{+}\left(0, S_{0}\right)$ and $\left(D_{s} V\left(t, S_{t}\right)\right)_{t}$ is a superreplicating portfolio, which is also Markov. This concludes the proof.

\section{$5 \quad$ Examples and applications}

We now present some examples. Their order is not random: in fact they use the theory presented above in a progressive way. More precisely, the first example shows the results via an explicit formula; in the second one, $\Sigma$ is contained in $\operatorname{GL}(n, \mathbb{R})$, so the solution of the BSB equation is smooth; in the third, $\Sigma$ is not contained in $\operatorname{GL}(n, \mathbb{R})$ but Hypothesis 14 holds and we can apply the results of Section 4; finally in the fourth example we can not apply the theoretical results of this article but the BSB has an explicit smooth solution and the BSB strategy is superhedging.

\subsection{Margrabe's exchange option}

We consider Margrabe's exchange option (see [18]), whose final payoff is the following:

$$
h(s)=\left(s_{1}-\lambda s_{2}\right)^{+}
$$

We note that $h$ is convex, so we can apply the results of the previous section if Hypothesis 14-(ii) holds. In this case we can check the correctness of 14 -(ii) directly if $d=2$ : in fact, denoting by $\gamma_{1}$ and $\gamma_{2}$ the rows of the generic volatility vector $\gamma \in \Sigma$, Hypothesis 14-(ii) implies that $\Gamma=\max _{\gamma \in \Sigma}\left\|\gamma_{1}-\gamma_{2}\right\|_{\mathbb{R}^{2}}>0$, so there exists an explicit solution to the BSB equation, given by

$$
V\left(t, s_{1}, s_{2}\right)=s_{1} N\left(d_{1}\right)-\lambda s_{2} N\left(d_{2}\right)
$$


where

$$
d_{1}=\frac{1}{\Gamma \sqrt{T-t}} \ln \left(\frac{s_{1}}{\lambda s_{2}}\right)+\frac{1}{2} \Gamma \sqrt{T-t}, \quad d_{2}=d_{1}-\Gamma \sqrt{T-t}
$$

and $N$ is the cumulative distribution function of a standard Gaussian random variable:

$$
N(d)=\frac{1}{\sqrt{2 \pi}} \int_{-\infty}^{d} e^{-\frac{x^{2}}{2}} d x
$$

(see [20] for details). Since $V \in C^{1,2}$, we can apply Theorem 5 directly.

Margrabe's exchange option is a rare case in which there is an explicit solution to the BSB equation. In the general case we are not so lucky and we have to use the theory of Sections 3 and 4 .

\subsection{RiskMetrics' stochastic volatility}

This example is a generalization of a stochastic volatility model proposed in [27]. We consider a model in which we have a pathwise constant volatility of the type

$$
\sigma_{t}=\sigma_{0} \mathbf{1}_{t<t_{0}}+\sum_{i=1}^{k} \sigma_{i} \mathbf{1}_{t_{i-1} \leq t<t_{i}}+\sigma_{t_{k}} \mathbf{1}_{t_{k} \leq t}
$$

where $t_{i}$ are fixed instants in $[0, T]$ and $\sigma_{1}, \ldots, \sigma_{k}$ are discrete random variables taking values in $\operatorname{GL}(n, \mathbb{R})$ independent of $W$ and such that $\sigma_{i}$ is $\mathcal{F}_{t_{i}}$-measurable for all $i=1, \ldots, k$. With these assumptions, Hypothesis 14-(ii) holds. Moreover, since $\sigma$ assumes values in $\operatorname{GL}(n, \mathbb{R})$, thanks to the results of Section 3, the BSB equation has a smooth solution for all final payoffs that are continuous and with linear growth.

As we have seen in Example 12, it is not always the case that $\Sigma$ is contained in $\operatorname{GL}(n, \mathbb{R})$, as the next example also shows.

\subsection{Markov stochastic correlation}

We consider a model in which there are two assets driven by the 2-dimensional Brownian motion $\left(W^{1}, W^{2}\right)$ and $\Sigma$ is as in Example 12. Moreover we assume that the correlation $\rho$ is given by $\rho_{t}=\frac{2}{\pi} \arctan Z_{t}$, where $Z$ is an Ornstein-Uhlenbeck process independent of $\left(W^{1}, W^{2}\right)$ having the dynamics

$$
d Z_{t}=\left(a-b Z_{t}\right) d t+\sigma_{3} d W_{t}^{3}
$$

with $W^{3}$ independent of $\left(W^{1}, W^{2}\right)$. Then $Z_{t}$ has a Gaussian law $N\left(\mu_{Z_{t}}, \sigma_{Z_{t}}^{2}\right)$, where $\mu_{Z_{t}}=$ $e^{-b t} Z_{0}+a\left(1-e^{-b t}\right) / b$ and $\sigma_{Z_{t}}^{2}=\sigma_{3}^{2}\left(1-e^{-2 b t}\right) / 2 b$. In this model, $\rho$ assumes values in $(-1,1)$ and the BSB equation is not uniformly parabolic. Moreover, the $\rho$ realizing the supremum is equal to +1 or -1 depending on the sign of $\frac{\partial V}{\partial s_{1} \partial s_{2}}$, so it does not seem easy to obtain an explicit solution. However we can use the results of Section 4 if we prove that Hypothesis 14 (ii) holds. This is equivalent to proving that the random variables

$$
\begin{aligned}
Y_{t} & =\frac{1}{\sigma_{1}}\left(\log \frac{S_{t}}{S_{0}}+\frac{1}{2} \sigma_{1}^{2} t\right)=W_{t}^{1} \\
Y_{t} & =\frac{1}{\sigma_{2}}\left(\log \frac{S_{t}}{S_{0}}+\frac{1}{2} \sigma_{2}^{2} t\right)=\rho_{t} W_{t}^{1}+\sqrt{1-\rho_{t}^{2}} W_{t}^{2}
\end{aligned}
$$


have a joint density with respect to the 2-dimensional Lebesgue measure. In order to see this, we take a generic $f \in C_{b}\left(\mathbb{R}^{2}\right)$ and calculate

$$
\begin{aligned}
\mathbb{E} & {\left[f\left(Y_{t}^{1}, Y_{t}^{2}\right)\right]=\mathbb{E}\left[f\left(W_{t}^{1}, \rho_{t} W_{t}^{1}+\sqrt{1-\rho_{t}^{2}} W_{t}^{2}\right)\right]=} \\
& =\int_{\mathbb{R}^{3}} f\left(x_{1}, \frac{2}{\pi} x_{1} \arctan x_{3}+x_{2} \sqrt{1-\left(\frac{2}{\pi} \arctan x_{3}\right)^{2}}\right) e^{-\frac{x_{1}^{2}+x_{2}^{2}}{2 t}-\frac{\left(x_{3}-\mu_{Z_{t}}\right)^{2}}{2 \sigma_{Z_{t}}^{2}}} d x
\end{aligned}
$$

By making the change of variables

$$
\begin{aligned}
y_{1} & =x_{1} \\
y_{2} & =\frac{2}{\pi} x_{1} \arctan x_{3}+x_{2} \sqrt{1-\left(\frac{2}{\pi} \arctan x_{3}\right)^{2}} \\
y_{3} & =x_{3}
\end{aligned}
$$

which is $C^{1}$ and invertible, we arrive at

$$
\mathbb{E}\left[f\left(Y_{t}^{1}, Y_{t}^{2}\right)\right]=\int_{\mathbb{R}^{3}} f\left(y_{1}, y_{2}\right) \phi\left(y_{1}, y_{2}\right) d y
$$

where $\phi$ is defined by

$$
\begin{aligned}
\phi\left(y_{1}, y_{2}\right)= & \int_{\mathbb{R}} \exp \left(-\frac{y_{1}^{2}}{2 t}-\frac{1}{2 t}\left(\frac{y_{2}-\frac{2}{\pi} y_{1} \arctan x_{3}}{\sqrt{1-\left(\frac{2}{\pi} \arctan y_{3}\right)^{2}}}\right)^{2}-\frac{\left(x_{3}-\mu_{Z_{t}}\right)^{2}}{2 \sigma_{Z_{t}}^{2}}\right) \times \\
& \times\left(1-\left(\frac{2}{\pi} \arctan y_{3}\right)^{2}\right)^{-1 / 2} d y_{3}
\end{aligned}
$$

In particular we obtain that $\left(Y^{1}, Y^{2}\right)$ has a density with respect to the Lebesgue measure, so Hypothesis 14-(ii) holds. This means that within this model we can obtain a Markov superstrategy using the results of Section 4 for every payoff $h$ satisfying Hypothesis 14- $(i)$ (to cite some examples, call options on the maximum of several assets and call and put options on the arithmetic mean).

The preceding example shows that the picture is still not complete. In fact, even BSB equations which are not uniformly parabolic and with non-convex final condition can have smooth solutions, as our final example shows.

\subsection{An example of non-convex payoff}

We consider an option whose final payoff is the following:

$$
h(s)=\left(\sqrt{s_{1} s_{2}}-K\right)^{+}
$$

This payoff can be used as a lower bound for options on the sum of 2 assets (see [9] for details). We suppose that we superhedge this option using a model in which $\Sigma$ is as in Example 12. In this case, $\Sigma$ is not contained in $\operatorname{GL}(n, \mathbb{R})$, so we can not use the results in Section 3 to conclude that the BSB equation has a smooth solution. Moreover $h$ is not 
convex, so neither can we apply the results of Section 4. However in this case there exists an explicit smooth solution to the BSB equation, given by

$$
V\left(t, s_{1}, s_{2}\right)=e^{\mu(T-t)} \sqrt{s_{1} s_{2}} N\left(d_{1}\right)-K N\left(d_{2}\right)
$$

where (using the notations of Example 12)

$\mu=-\frac{1}{8}\left(\sigma_{1}-\sigma_{2}\right)^{2}, \quad d_{1}=\frac{1}{\tilde{\gamma}_{t}} \ln \frac{\sqrt{S_{1}(t) S_{2}(t)}}{K e^{-\mu(T-t)}}+\frac{1}{2} \tilde{\gamma}_{t}, \quad d_{2}=d_{1}-\tilde{\gamma}_{t}, \quad \tilde{\gamma}_{t}=\frac{1}{2}\left|\sigma_{1}+\sigma_{2}\right| \sqrt{T-t}$

(see [20] for details).

\section{References}

[1] Avellaneda M, Levy A, Parás A (1995) Pricing and hedging derivative securities in markets with uncertain volatilities. Applied Mathematical Finance 2: 73-88

[2] Bardi M, Capuzzo Dolcetta I (1997) Optimal control and viscosity solutions of Hamilton-Jacobi-Bellman equations. Birkhauser

[3] Barucci E, Gozzi F, Vespri V (1999) A Semigroup Approach to No-Arbitrage Pricing Theory. In: Dalang R, Dozzi M, Russo F (ed.) Seminar on Stochastic Analysis, Random Fields and Applications, Centro Stefano Franscini, Ascona, September 1996. Birkhäuser, pp. 1-14

[4] Black F, Scholes M (1973) The pricing of options and corporate liabilities. Journal of Political Economy 81: 637-659

[5] Crandall MG, Kocan M, and Swięch A (2000) $L^{p}$-Theory for fully nonlinear uniformly parabolic equations. Communications in Partial Differential Equations 25: 1997-2053

[6] Cvitanic J, Pham H, Touzi N (1999) Super-replication in stochastic volatility models under portfolio constraints. Advances in Applied Probability 36: 523-545

[7] Dewynne J, Howison S, Wilmott P (1993) Option Pricing: Mathematical Models and Computation. Oxford Financial Press, Oxford

[8] El Karoui N, Geman H, Rochet JC (1995) Changes of numeraire, changes of probability measures and option pricing. Journal of Applied Probability 32: 443-458.

[9] El Karoui N, Jeanblanc M, Shreve SE (1998) Robustness of the Black and Scholes formula. Math. Finance 8: 93-126.

[10] El Karoui N, Quenez MC (1995) Dynamic programming and pricing contingent claims in incomplete markets. SIAM Journal of Control and Optimization 33: 29-66

[11] Fleming WH, Soner HM (1993) Controlled Markov processes and viscosity solutions. Springer

[12] Gozzi F, Monte R, Vespri V (2001) Generation of analytic semigroups for degenerate elliptic operators arising in financial mathematics. to appear in Advances in Differential Equation 
[13] Johnson H (1987) Options on the maximum or the minimum of several assets. Journal of Financial and Quantitative Analysis 22: 277-283

[14] Krylov NV (1980) Controlled diffusion processes. Springer

[15] Lamberton D, Lapeyre B (1991) Introduction au calcul stochastique appliquée à la Finance. Société de Mathématiques Appliquées et Industrielles

[16] Lions PL (1982) Generalized solutions of Hamilton-Jacobi equations. Pitman Advanced Publishing Program

[17] Lyons TJ (1995) Uncertain volatility and the risk-free synthesis of derivatives. Applied Mathematical Finance 2: 117-133

[18] Margrabe W (1978) The price to exchange an asset for another. The Journal of Finance 1: $177-186$

[19] Martini C (2000) On the marginal laws of one-dimensional stochastic integrals with uniformly elliptic integrand. Annales de l'Institut Henry Poincarè 36: 35-43

[20] Romagnoli S, Vargiolu T (2000) Robustness of the Black-Scholes formula in the case of options on several assets. Finance and Stochastics 4: 325-341

[21] Soner HM, Touzi N (2000) Superreplication under Gamma constraints. SIAM J. Control Optimization 39: 73-96

[22] Stulz RM (1982) Options on the minimum or the maximum of two risky assets. Journal of Financial Economics 10: 161-185

[23] Vargiolu T (2001) Existence, uniqueness and smoothness for the Black-ScholesBarenblatt equation. University of Padova, Department of Pure and Applied Mathematics, Rapporto Interno n. 5, http://www.math.unipd.it/ ${ }^{\sim}$ vargiolu/BSB.pdf

[24] Wang L (1992) On the regularity theory of fully nonlinear parabolic equations: I, II. Communications on Pure and Applied Mathematics, 45: 27-76 and 141-178

[25] Yong J, Zhou XY (1999) Stochastic Control: Hamiltonian Systems and HJB Equations. Springer

[26] Yosida K (1980) Functional Analysis. Springer

[27] Zangari P (1996) An improved methodology for measuring VaR. RiskMetrics Monitor 2: $7-25$ 